\title{
Existence of Solutions to Boundary Value Problems for a Class of Nonlinear Fuzzy Fractional Differential Equations
}

\author{
Yupin Wang*, Shurong Sun and Zhenlai Han \\ School of Mathematical Sciences, University of Jinan, Jinan, Shandong 250022, P R China \\ Email: wangyupin8252@163.com
}

\begin{abstract}
In this paper, we investigate the existence and uniqueness of solution to boundary value problems for a class of nonlinear fuzzy factional differential equations involving the fuzzy $g H$ fractional Caputo derivative. By means of the Schauder fixed point theorem in semi-linear spaces and integral inequality technique, some qualitative results of solutions are obtained. An example is provided from which new results are found.
\end{abstract}

Keywords: Fuzzy fractional differential equations, generalized Hukuhara differentiability, boundary value problems, Schauder fixed point theorem, generalized Gronwall inequality.

\section{Introduction}

The study of fuzzy differential equations forms a suitable setting for the mathematical modeling of real world problems in which uncertainty or vagueness pervades. As from some concepts of generalized fuzzy derivative begin to emerge in the mid-2000s, this field has developed rapidly and attracted much attention of many scholars. In 2013, Khastan et al. studied the existence of solutions to a class of first-order linear fuzzy differential equations subject to periodic boundary conditions (cf. [1]). In 2016, Wang studied two-point boundary value problems for a class of first-order nonlinear fuzzy differential equations (cf. [2]). Nowadays, the theory of fuzzy differential equations could be applicable in many areas, for instance, physics, thermodynamics, biology, medicine, chemistry and many other fields of science (see e. g., $[3,4,5,6,7]$ and references contained therein).

In 2010, the concept of the solution to fuzzy fractional differential equations (FFDEs) was proposed by Agarwal et al. (cf. [8]). In 2013, the classical Schauder fixed point theorem has been extended to semi-linear Banach spaces and the authors discussed the existence and uniqueness of solution to the following integral equations

$$
u(t)=u_{0}(t) \oplus \mathfrak{I}^{q} f(t, u(t)), \quad 0<q<1,
$$

which provides a new way to consider FFDEs because semi-linear metric space is a generalization of fuzzy number space (cf. [9]). The establishment of the basic theory of semi-linear metric space constructs a new framework for fuzzy analysis. In 2014, Khastan et al. (cf. [10]) utilized the generalized Schauder fixed point theorem to study the existence of solutions to FFDEs

$$
\mathfrak{D}^{q} u(t)=f(t, u(t)), \quad 0<q<1
$$

Meanwhile, many experts have made a great number of outstanding contributions in this vigorous and interesting field under generalized Hukuhara differentiability. We can refer to papers $[11,12,13,14,15]$ etc.

Boundary value problems for FFDEs, which are the combination of fuzzy analysis and boundary value problems for fractional differential equations, are a very magical and practical field. However, the works above are curbed in the order $q \in(0,1]$ as far as we know. In this paper, we initiate to consider the existence and uniqueness of solution to a class of FFDEs

$$
{ }_{g H}^{C} \mathfrak{D}_{*}^{q} u(t)=f(t, u(t)), \quad 0<t<T
$$

subject to boundary conditions

$$
u(0)=\lambda u(T), u_{g H}^{\prime}(0)=u_{0} \in \mathbb{E}_{c}^{1},
$$


where ${ }_{g H}^{C} \mathfrak{D}_{*}^{q}$ is the fuzzy $g H$-fractional Caputo derivative, $q \in(1,2]$ is a real number, $f:[0, T] \times \mathbb{E}_{c}^{1} \rightarrow$ $\mathbb{E}_{c}^{1} \backslash \mathbb{R}$ is a continuous and compact fuzzy-valued function and $\lambda \in(0,1) \cup(1,+\infty)$ is a parameter. We utilize Schauder fixed point theorem in semi-linear spaces to study the existence of solutions to boundary value problems (1.1)-(1.2). This work is motivated by papers $[2,9,10]$. Most of all, this may be the first time to consider such problem about "higher order" fuzzy fractional differential equations.

The remainder of this paper is organized as follows. In Section 2, we collect some basic definitions, properties and lemmas about fuzzy set theory and fractional calculus theory. In Section 3, we investigate the existence and uniqueness of solution to boundary value problems (1.1)-(1.2). In Section 4, an example is given to illustrate our main results. In Section 5, conclusion and some recommendations for future work are drawn.

\section{Preliminaries}

For convenience, we give some definitions and introduce the necessary notations and lemmas (see e.g., monographs $[16,17,18,19]$ and references contained therein) which will be used in the following sections.

Let $\mathbb{E}^{1}$ be the space of all fuzzy sets in $\mathbb{R}$, that is, $\mathbb{E}^{1}$ is the space of all functions $u: \mathbb{R} \rightarrow[0,1]$ satisfying the following conditions:

(i) $u$ is normal, i.e., there exists $x_{0} \in \mathbb{R}$, such that $u\left(x_{0}\right)=1$;

(ii) $[u]^{0}=\overline{\{x \in \mathbb{R} \mid u(x)>0\}}$ is compact;

(iii) $u$ is a convex fuzzy-valued function, i. e., $u\left(\lambda x_{1}+(1-\lambda) x_{2}\right) \geq \min \left\{u\left(x_{1}\right), u\left(x_{2}\right)\right\}$ for any $x_{1}, x_{2} \in \mathbb{R}$ and $\lambda \in(0,1)$;

(iv) $u$ is an upper semi-continuous function on $\mathbb{R}$, i. e., $u\left(x_{0}\right) \geq \lim _{x \rightarrow x_{0}^{+}} u(x)$ for any $x_{0} \in \mathbb{R}$.

Usually, $\mathbb{E}^{1}$ is also called the space of fuzzy numbers. And $\mathbb{R} \subseteq \mathbb{E}^{1}$ if real number set $\mathbb{R}$ is understood as $\mathbb{R}=\left\{\chi_{\{x\}} \mid x\right.$ is usual real number $\}$.

Define $\underline{u}$ and $\bar{u}$ respectively as the lower and upper branches of the fuzzy set $u \in \mathbb{E}^{1}$, respectively. The set

$$
[u]^{\alpha}=\{x \in \mathbb{R} \mid u(x) \geq \alpha\}:=\left[\underline{u}^{\alpha}, \bar{u}^{\alpha}\right]
$$

is called the $\alpha$-level set of the fuzzy set $u$, where $\alpha \in(0,1]$. From (i)-(iv), it follows that the $\alpha$-level set $[u]^{\alpha} \in K_{c}(\mathbb{R})$ for all $\alpha \in[0,1]$, where $K_{c}(\mathbb{R})$ is the family of all nonempty, compact and convex subsets of $\mathbb{R}$.

The following operations, which are based on a generalized Zadeh's extension principle, define a semi-linear structure on $\mathbb{E}^{1}$ :

$$
\begin{aligned}
& -(u \oplus v)(x)=\sup _{x_{1}+x_{2}=x} \min \left\{u\left(x_{1}\right), v\left(x_{2}\right)\right\} \\
& -(\lambda u)(x)= \begin{cases}u(x / \lambda), & \lambda \neq 0, \\
\chi_{\{0\}}, & \lambda=0,\end{cases}
\end{aligned}
$$

where $u, v \in \mathbb{E}^{1}, \lambda \in \mathbb{R}$. The $\alpha$-level set of fuzzy sets satisfies the following properties (see [17]):

$$
\begin{aligned}
& -[u \oplus v]^{\alpha}=[u]^{\alpha}+[v]^{\alpha}=\left[\underline{u}^{\alpha}+\underline{v}^{\alpha}, \bar{u}^{\alpha}+\bar{v}^{\alpha}\right] ; \\
& -[\lambda u]^{\alpha}=\lambda[u]^{\alpha}= \begin{cases}{\left[\lambda \underline{u}^{\alpha}, \lambda \bar{u}^{\alpha}\right],} & \lambda \geq 0, \\
{\left[\lambda \bar{u}^{\alpha}, \lambda \underline{u}^{\alpha}\right],} & \lambda<0 .\end{cases}
\end{aligned}
$$

Here, $[u]^{\alpha}+[v]^{\alpha}$ means the usual addition of two subsets of $\mathbb{R}$ and $\lambda[u]^{\alpha}$ means the usual product between a scalar and a subset of $\mathbb{R}$.

Lemma 2.1. [20]

(i) If we denote $\widehat{0}=\chi_{\{0\}}$ then $\widehat{0} \in \mathbb{E}^{1}$ is neutral element with respect to $\oplus$, i. e., $u \oplus \widehat{0}=\widehat{0} \oplus u=u$, for all $u \in \mathbb{E}^{1}$

(ii) With respect to $\widehat{0}$, none of $u \in \mathbb{E}^{1} \backslash \mathbb{R}$ has inverse in $\mathbb{E}^{1}$ (with respect to $\oplus$ ); 
(iii) For any $a, b \in \mathbb{R}$, with $a, b \geq 0$ or $a, b \leq 0$ and any $u \in \mathbb{E}^{1}$, we have $(a+b) u=a u \oplus$ bu. For general $a, b \in \mathbb{R}$, the above property does not hold;

(iv) For any $\lambda \in \mathbb{R}$ and any $u, v \in \mathbb{E}^{1}$, we have $\lambda(u \oplus v)=\lambda u \oplus \lambda v$;

(v) For any $\lambda, \mu \in \mathbb{R}$ and any $u, \in \mathbb{E}^{1}$, we have $\lambda(\mu u)=(\lambda \mu) u$.

Lemma 2.2. Suppose that $\lambda \in \mathbb{R}, w \in \mathbb{E}^{1} \backslash \mathbb{R}$, then

$$
u=\lambda(u \oplus w)
$$

and

$$
v=\lambda v \oplus w
$$

have solutions in $\mathbb{E}^{1} \backslash \mathbb{R}$ if and only if $\lambda \in(-1,1)$. In addition,

$$
u=\left\{\begin{array}{ll}
\frac{\lambda}{1-\lambda} w, & \lambda \in[0,1), \\
\frac{\lambda^{2}}{1-\lambda^{2}} w \oplus \frac{\lambda}{1-\lambda^{2}} w, & \lambda \in(-1,0)
\end{array} \quad \text { and } v= \begin{cases}\frac{1}{1-\lambda} w, & \lambda \in[0,1), \\
\frac{1}{1-\lambda^{2}} w \oplus \frac{\lambda}{1-\lambda^{2}} w, & \lambda \in(-1,0) .\end{cases}\right.
$$

Proof. Necessity has been given by [2, Lemma 2]. Here we only discuss the sufficiency. On the one hand, set $u_{1}=\frac{\lambda}{1-\lambda} w \in \mathbb{E}^{1} \backslash \mathbb{R}$ when $\lambda \in[0,1)$. From Lemma 2.1, for the right-hand side of fuzzy algebraic equation (2.1), we have

$$
\lambda\left(u_{1} \oplus w\right)=\lambda\left(\frac{\lambda}{1-\lambda} w \oplus w\right)=\frac{\lambda^{2}}{1-\lambda} w \oplus \lambda w=\frac{\lambda}{1-\lambda} w=u_{1},
$$

which means $u_{1}$ is a solution to equation (2.1) when $\lambda \in[0,1)$. On the other hand, let $u_{2}=\frac{\lambda^{2}}{1-\lambda^{2}} w \oplus \frac{\lambda}{1-\lambda^{2}} w$ when $\lambda \in(-1,0)$. For the right-hand side of equation (2.1), we have

$$
\lambda\left(u_{2} \oplus w\right)=\frac{\lambda^{3}}{1-\lambda^{2}} w \oplus \frac{\lambda^{2}}{1-\lambda^{2}} w \oplus \lambda w=\frac{\lambda}{1-\lambda^{2}} w \oplus \frac{\lambda^{2}}{1-\lambda^{2}} w=u_{2},
$$

which means $u_{2}$ is a solution to equation $(2.1)$ when $\lambda \in(-1,0)$. Therefore, equation (2.1) has solution when $\lambda \in(-1,1)$. As a similar argument as above, we can discuss fuzzy algebraic equation (2.2). So we omit here. The proof is completed.

As a generalization of Hausdorff-Pompeiu metric on compact and convex set, a metric $d$ on $\mathbb{E}^{1}$ can be defined by

$$
d(u, v)=\sup _{\alpha \in[0,1]} d_{H}\left([u]^{\alpha},[v]^{\alpha}\right)=\sup _{\alpha \in[0,1]} \max \left\{\left|\underline{u}^{\alpha}-\underline{v}^{\alpha}\right|,\left|\bar{u}^{\alpha}-\bar{v}^{\alpha}\right|\right\}
$$

where $u, v \in \mathbb{E}^{1}$ and $d_{H}$ is the Hausdorff-Pompeiu metric.

Definition 2.1. [21] Let $u, v \in \mathbb{E}^{1}$. If there exists unique fuzzy number $w \in \mathbb{E}^{1}$ such that $v \oplus w=u$, then $w$ is called the $H$-difference of $u$ and $v$, which is denoted $u \ominus v$.

Lemma 2.3. [17,18] If $u, v, x, y \in \mathbb{E}^{1}$, then

(i) $d(u \oplus x, v \oplus x)=d(u, v)$;

(ii) $d(\lambda u, \lambda v)=|\lambda| d(u, v), \lambda \in \mathbb{R}$;

(iii) $d(u \oplus x, v \oplus y) \leq d(u, v)+d(x, y)$;

(iv) $d(\lambda u, \mu u)=|\lambda-\mu| d(u, \widehat{0}), \lambda, \mu \geq 0$;

(v) $d(u \ominus x, v \ominus y) \leq d(u, v)+d(x, y)$, provided the differences $u \ominus x$ and $v \ominus y$ exist;

(vi) $\left(\mathbb{E}^{1}, d\right)$ is a complete metric space.

Denote $\mathbb{E}_{c}^{1}$ as the space of fuzzy number $u \in \mathbb{E}^{1}$ with the property that the function $\alpha \rightarrow[u]^{\alpha}$ is continuous in the meaning of the Hausdorff-Pompeiu metric on [0,1]. According to Theorem 3.1 in [22], we know $\left(\mathbb{E}_{c}^{1}, d\right)$ is a complete metric space. Let $C^{\mathbb{E}}[a, b]$ denote a space of fuzzy-valued functions which are continuous on $[a, b]$. Then $\left(C^{\mathbb{E}}\left([a, b], \mathbb{E}_{c}^{1}\right), D\right)$ is a complete metric space, where

$$
D(u, v):=\sup _{t \in[0,1]} d(u(t), v(t)) .
$$

Next, we list some definitions and lemmas about relative compactness in $\mathbb{E}_{c}^{1}$. 
Definition 2.2. [22] A subset $A \subseteq \mathbb{E}_{c}^{1}$ is said to be compact-supported if there exists a compact set $K \subseteq \mathbb{R}$ such that $[y]^{0} \subseteq K$ for all $y \in A$.

Definition 2.3. [22] A subset $A \subseteq \mathbb{E}_{c}^{1}$ is said to be level-equicontinuous at $\alpha_{0} \in[0,1]$ if for all $\varepsilon>0$, there exists $\delta>0$ such that $\left|\alpha-\alpha_{0}\right|<\delta$ implies $d_{H}\left([y]^{\alpha},[y]^{\alpha_{0}}\right)<\varepsilon$, for all $y \in A$.

$A$ is level-equicontinuous on $[0,1]$ if $A$ is level-equicontinuous at $\alpha$ for all $\alpha \in[0,1]$.

Lemma 2.4. (Arzelá-Ascoli Theorem)[22, Theorem 4.1] Let $M$ be a compact metric space, $N$ be a metric space and $E \subseteq C^{\mathbb{E}}(M, N)$. Then $E$ is relatively compact if and only if

(i) E equicontinuous;

(ii) For every $a \in M$, the set $E(a)=\{f(a) \mid f \in E\}$ is relatively compact in $N$.

Lemma 2.5. [22, Theorem 4.5] Let $A$ be a compact-supported subset of $\mathbb{E}_{c}^{1}$. Then the following assertions are equivalent:

(a) $A$ is a relatively compact subset of $\left(\mathbb{E}_{c}^{1}, d\right)$;

(b) $A$ is level-equicontinuous on $[0,1]$.

Definition 2.4. [10] A continuous function $f:[0,1] \times \mathbb{E}_{c}^{1} \rightarrow \mathbb{E}_{c}^{1}$ is said to be compact if for every subinterval $I \subseteq[0,1]$ and every bounded subset $A \subseteq \mathbb{E}_{c}^{1}$, then $f(I \times A)$ is relatively compact in $\left(\mathbb{E}_{c}^{1}, d\right)$.

Definition 2.5. [17, Definition 2.4.1] The Aumann integral of a fuzzy-valued function $F: I \rightarrow \mathbb{E}^{1}$ over $I$ is defined levelwise

$$
\left[\int_{I} F(t) d t\right]^{\alpha}=\int_{I} F_{\alpha}(t) d t=\left\{\int_{I} g(t) d t: g \in S\left(F_{\alpha}\right)\right\}
$$

for all $\alpha \in(0,1]$, where $S\left(F_{\alpha}\right)$ is the subset of all integrable selections of set-valued mapping $F_{\alpha}$.

Lemma 2.6. [23] Let $F, G: I \rightarrow \mathbb{E}^{1}$ be integrable and $\lambda \in \mathbb{R}$. Then

(i) $\int_{I}(F \oplus G)=\int_{I} F \oplus \int_{I} G$;

(ii) $\int_{I}(\lambda F)=\lambda \int_{I} F$; (iii) $d(F, G)$ is integrable on $I$;

(iv) $d\left(\int_{I} F, \int_{I} G\right) \leq \int_{I} d(F, G)$.

Definition 2.6. [10, Definition 2.10] Let $F \in C^{\mathbb{E}}(a, b] \cap L^{\mathbb{E}}(a, b]$. The fuzzy fractional integral of order $q>0$ of $F$ is defined as

$$
\mathfrak{I}^{q} F(t)=\frac{1}{\Gamma(q)} \int_{a}^{t}(t-s)^{q-1} F(s) d s, t \in(a, b)
$$

provided that the integral in the right-hand side is pointwise well-defined. For $q=1$ we obtain $\mathfrak{I}^{1} F(t)=$ $\int_{a}^{t} F(s) d s$, that is, the classical integral operator.

Lemma 2.7. [24] Let $F:[a, b] \rightarrow \mathbb{E}^{1}$ be an integrable fuzzy function and $p, q>0$, then

$$
\mathfrak{I}^{p} \mathfrak{I}^{q} F(t)=\mathfrak{I}^{p+q} F(t)
$$

Definition 2.7. [25,26] Given two fuzzy numbers $u, v \in \mathbb{E}^{1}$, the generalized Hukuhara difference ( $g H$-difference for short) is the fuzzy number $w$, if it exists, such that

$$
u \ominus_{g H} v=w \Longleftrightarrow\left\{\begin{aligned}
\text { (i) } & u=v \oplus w \\
\text { or (ii) } & v=u \oplus(-1) w .
\end{aligned}\right.
$$

It is easy to show that (i) and (ii) are both valid if and only if $w$ is a crisp number. 
Definition 2.8. [26] Let $t_{0} \in(a, b)$ and $h$ be such that $t_{0}+h \in(a, b)$. Then the generalized Hukuhara derivative of a function $F:(a, b) \rightarrow \mathbb{E}^{1}$ at $t_{0}$ is defined as

$$
F_{g H}^{\prime}\left(t_{0}\right)=\lim _{h \rightarrow 0} \frac{1}{h}\left[F\left(t_{0}+h\right) \ominus_{g H} F\left(t_{0}\right)\right] .
$$

If $F_{g H}^{\prime}\left(t_{0}\right) \in \mathbb{E}^{1}$ satisfying (2.3) exists, we say that $F$ is generalized Hukuhara differentiable $(g H$ differentiable for short) at $t_{0}$.

Remark 2.1. Let $F:[a, b] \rightarrow \mathbb{E}^{1}$. One says $F$ is twice $g H$-differentiable at $t_{0} \in(a, b)$, if $F_{g H}^{\prime}(t)$ exists on a neighborhood of $t_{0}$ as a fuzzy-valued function and it is $g H$-differentiable at $t_{0}$. Similarly, we can define higher order $g H$-derivatives of fuzzy-valued function.

Definition 2.9. [26] Let $F:[a, b] \rightarrow \mathbb{E}^{1}$ and $t_{0} \in(a, b)$ with $\underline{F}^{\alpha}(t)$ and $\bar{F}^{\alpha}(t)$ both differentiable at $t_{0}$. We say that $F$ is (i)- $g H$-differentiable at $t_{0}$ if

$$
\left[F_{g H}^{\prime}\left(t_{0}\right)\right]^{\alpha}=\left[\left(\underline{F}^{\alpha}\right)^{\prime}\left(t_{0}\right),\left(\bar{F}^{\alpha}\right)^{\prime}\left(t_{0}\right)\right], 0 \leq \alpha \leq 1,
$$

and $F$ is (ii)- $g H$-differentiable at $t_{0}$ if

$$
\left[F_{g H}^{\prime}\left(t_{0}\right)\right]^{\alpha}=\left[\left(\bar{F}^{\alpha}\right)^{\prime}\left(t_{0}\right),\left(\underline{F}^{\alpha}\right)^{\prime}\left(t_{0}\right)\right], 0 \leq \alpha \leq 1 .
$$

Definition 2.10. [11, Definition 3.1] Let $F_{g H}^{(m)} \in C^{\mathbb{E}}[a, b] \cap L^{\mathbb{E}}[a, b]$. The fuzzy $g H$-fractional Caputo differentiability of fuzzy-valued function $F$ is defined as following:

$$
\left(\begin{array}{l}
C \\
g H
\end{array} \mathfrak{D}_{*}^{q} F\right)(t)=\left(\mathfrak{I}^{m-q} F_{g H}^{(m)}\right)(t)=\frac{1}{\Gamma(m-q)} \int_{a}^{t}(t-s)^{m-q-1} F_{g H}^{(m)}(s) d s,
$$

where $m-1<q \leq m, m \in \mathbb{N}_{+}$and $t \geq a$.

Definition 2.11. [11, Definition 3.4] Let $F:[a, b] \rightarrow \mathbb{E}^{1}$ be fuzzy $g H$-fractional Caputo differentiable at $t_{0} \in(a, b)$. We say that $F$ is ${ }^{C F}[(\mathrm{i})-g H]$-differentiable at $t_{0}$ if

$$
\left[\left(\begin{array}{l}
C \\
g H
\end{array} \mathfrak{D}_{*}^{q} F\right)\left(t_{0}\right)\right]^{\alpha}=\left[\left({ }^{C} D_{*}^{q} \underline{F}^{\alpha}\right)\left(t_{0}\right),\left({ }^{C} D_{*}^{q} \bar{F}^{\alpha}\right)\left(t_{0}\right)\right], 0 \leq \alpha \leq 1,
$$

and $F$ is $C F\left[(\right.$ ii) $-g H]$-differentiable at $t_{0}$ if

$$
\left[\left(\begin{array}{l}
C \\
g H
\end{array} \mathfrak{D}_{*}^{q} F\right)\left(t_{0}\right)\right]^{\alpha}=\left[\left({ }^{C} D_{*}^{q} \bar{F}^{\alpha}\right)\left(t_{0}\right),\left({ }^{C} D_{*}^{q} \underline{F}^{\alpha}\right)\left(t_{0}\right)\right], 0 \leq \alpha \leq 1,
$$

where ${ }^{C} D_{*}^{q}$ is the Caputo fractional derivative of a real-valued function.

According to Definition 2.10, we can get the following lemma immediately.

Lemma 2.8. Let $F$ have $\lceil q\rceil$ th-order Caputo $g H$-derivative and $q>1$, where $\lceil q\rceil$ denotes the ceiling function of the number $q$. Then

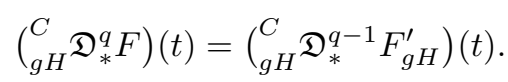

Remark 2.2. From the lemma above, it is not difficult to see $\left(\begin{array}{l}C H \\ g H\end{array} \mathfrak{D}_{*}^{q} F_{g H}^{(m)}\right)(t)=\left({ }_{g H}^{C} \mathfrak{D}_{*}^{q+m} F\right)(t)$ if $F_{g H}^{(m)}(t)$ and $\left({ }_{g H}^{C} \mathfrak{D}_{*}^{q+m} F\right)(t)$ exist for $m \in \mathbb{N}$ and $q \in \mathbb{R}_{+}$. But, it is not always true for the case $\left({ }_{g H}^{C} \mathfrak{D}_{*}^{q_{1}} \cdot{ }_{g H}^{C} \mathfrak{D}_{*}^{q_{2}} F\right)(t)=\left({ }_{g H}^{C} \mathfrak{D}_{*}^{q_{1}+q_{2}} F\right)(t)$ where $q_{1}, q_{2} \in \mathbb{R}_{+}$, even $\left.{ }_{g H^{C}}^{C} \mathfrak{D}_{*}^{q_{1}+q_{2}} F\right)(t)$ exists.

Lemma 2.9. [11] Let $0<q \leq 1$ and $t_{0} \in \mathbb{R}$, initial value problem

$$
\left\{\begin{array}{l}
\stackrel{C}{g H} \mathfrak{D}_{*}^{q} u(t)=f(t, u(t)) \\
u\left(t_{0}\right)=u_{0} \in \mathbb{E}^{1}
\end{array}\right.
$$

is equivalent to the following integral equation

$$
u(t) \ominus_{g H} u\left(t_{0}\right)=\frac{1}{\Gamma(q)} \int_{t_{0}}^{t}(t-s)^{q-1} f(s, u(s)) d s .
$$


Remark 2.3. In particular, two cases of existence of the generalized $H$-difference imply that equation (2.4) is actually a unified formulation of the following integral equations

$$
u(t)=u_{0} \oplus \frac{1}{\Gamma(q)} \int_{t_{0}}^{t}(t-s)^{q-1} f(s, u(s)) d s
$$

if $u(t)$ is $C F[(\mathrm{i})-g H]$-differentiable and

$$
u(t)=u_{0} \ominus \frac{-1}{\Gamma(q)} \int_{t_{0}}^{t}(t-s)^{q-1} f(s, u(s)) d s
$$

if $u(t)$ is $C F[(\mathrm{ii})-g H]$-differentiable.

Remark 2.4. Take $q=1$ in Lemma 2.9. Then we immediately get a similar result in integer order case.

Lemma 2.10. Let $1<q \leq 2$. Assume that $f:[0, T] \times \mathbb{E}^{1} \rightarrow \mathbb{E}^{1}$ is a continuous function. A fuzzy-valued function $u:[0, T] \rightarrow \mathbb{E}^{1}$ is a solution to the initial value problem

$$
\left\{\begin{array}{l}
{ }_{g H}^{C} \mathfrak{D}_{*}^{q} u(t)=f(t, u(t)) \\
u(0)=k_{1} \in \mathbb{E}^{1} \\
u_{g H}^{\prime}(0)=k_{2} \in \mathbb{E}^{1}
\end{array}\right.
$$

if and only if $u$ satisfies one of the following fuzzy integral equations:

$$
u(t)=k_{1} \oplus k_{2} t \oplus \frac{1}{\Gamma(q)} \int_{0}^{t}(t-s)^{q-1} f(s, u(s)) d s,
$$

where $u$ is (i)-gH-differentiable and $u_{g H}^{\prime}$ is ${ }^{C F}[(i)$-gH]-differentiable, or

$$
u(t)=k_{1} \oplus k_{2} t \ominus \frac{-1}{\Gamma(q)} \int_{0}^{t}(t-s)^{q-1} f(s, u(s)) d s,
$$

where $u$ is (i)-gH-differentiable and $u_{g H}^{\prime}$ is ${ }^{C F}[(i i)$-gH]-differentiable, or

$$
u(t)=k_{1} \ominus(-1)\left[k_{2} t \oplus \frac{1}{\Gamma(q)} \int_{0}^{t}(t-s)^{q-1} f(s, u(s)) d s\right],
$$

where $u$ is (ii)-gH-differentiable and $u_{g H}^{\prime}$ is ${ }^{C F}[(i)$-gH]-differentiable, or

$$
u(t)=k_{1} \ominus(-1)\left[k_{2} t \ominus \frac{-1}{\Gamma(q)} \int_{0}^{t}(t-s)^{q-1} f(s, u(s)) d s\right],
$$

where $u$ is (ii)-gH-differentiable and $u_{g H}^{\prime}$ is ${ }^{C F}[(i i)-g H]$-differentiable.

Proof. According to Lemma 2.8, equation (2.5) can be rewritten as

$$
\left(\begin{array}{l}
C \\
g H
\end{array} \mathfrak{D}_{*}^{q-1} u_{g H}^{\prime}\right)(t)=f(t, u(t)) .
$$

Without loss of generality, we only discuss the case that $u$ is (i)- $g H$-differentiable and $u_{g H}^{\prime}$ is $C F[(\mathrm{i})-g H]$ differentiable. The proofs of the other cases are similar.

Notice continuity of $f:[0, T] \times \mathbb{E}^{1} \rightarrow \mathbb{E}^{1}$ and $u:[0, T] \rightarrow \mathbb{E}^{1}$. According to Lemma 2.9 and Remark 2.3 , equation (2.5) with the condition (2.7) is equivalent to

$$
u_{g H}^{\prime}(t)=k_{2} \oplus \mathfrak{I}^{q-1} f(t, u(t))=k_{2} \oplus \frac{1}{\Gamma(q-1)} \int_{0}^{t}(t-s)^{q-2} f(s, u(s)) d s .
$$


From Remark 2.4 and initial condition (2.6), equation (2.9) is equivalent to

$$
u(t)=k_{1} \oplus k_{2} t \oplus \mathfrak{I}^{1} \mathfrak{I}^{q-1} f(t, u(t)) .
$$

According to Lemma 2.7, we get

$$
u(t)=k_{1} \oplus k_{2} t \oplus \mathfrak{I}^{q} f(t, u(t)),
$$

that is, equation (2.8). Conversely, condition $u_{g H}^{\prime}(0)=k_{2}$ is assured by equation (2.9). Meanwhile, equation (2.8) apparently satisfies (1.1) as well as condition (1.2). The proof is completed.

At the end of this part, we give Schauder fixed point theorem in semi-linear spaces and generalized Gronwall inequality, which are fundamental in the proofs of our main results.

Lemma 2.11. (Schauder fixed point theorem in semi-linear spaces)[9, Theorem 3.4] Let $B$ be a nonempty, closed, bounded and convex subset of a semi-linear Banach space $S$ having the cancellation property and suppose $P: B \rightarrow B$ is a compact operator. Then $P$ has at least one fixed point in $B$.

Lemma 2.12. (Generalized Gronwall inequality)[27, Theorem 1] Suppose $q>0, a(t)$ is a nonnegative function locally integrable on $0 \leq t<T$ (some $T \leq+\infty$ ) and $g(t)$ is a nonnegative, nondecreasing continuous function defined on $0 \leq t<T, g(t) \leq M$ (constant), and suppose $u(t)$ is nonnegative and locally integrable on $0 \leq t<T$ with

$$
u(t) \leq a(t)+g(t) \int_{0}^{t}(t-s)^{q-1} u(s) d s
$$

on this interval. Then

$$
u(t) \leq a(t)+\int_{0}^{t}\left[\sum_{n=1}^{\infty} \frac{(g(t) \Gamma(q))^{n}}{\Gamma(n q)}(t-s)^{n q-1} a(s)\right] d s, \quad 0 \leq t<T .
$$

\section{Main Results}

At the beginning of this section, we give integral representation of boundary value problems (1.1)-(1.2). Before this, we give the definition of solutions to these problems.

Definition 3.1. A function $u \in C^{\mathbb{E}}\left([0, T], \mathbb{E}_{c}^{1}\right)$ is said to be a $C F[(m-n)-g H]$-differentiable solution ( $m$ and $n$ can be taken as i or ii) to boundary value problems (1.1)-(1.2), if $u$ is $(m)$-gH-differentiable and $u_{g H}^{\prime}$ is $C F[(n)-g H]$-differentiable on the entire interval $[0, T]$ as well as $u$ satisfies $(1.1)-(1.2)$.

Lemma 3.1. A fuzzy-valued function $u:[0, T] \rightarrow \mathbb{E}_{c}^{1}$ is a solution to the problems (1.1)-(1.2) if and only if $u$ satisfies one of the following fuzzy integral equations:

$$
u(t)=\Lambda_{1} \oplus u_{0} t \oplus \frac{1}{\Gamma(q)} \int_{0}^{t}(t-s)^{q-1} f(s, u(s)) d s, \quad t \in[0, T]
$$

if $\lambda \in(0,1), u$ is (i)-gH-differentiable and $u_{g H}^{\prime}$ is $C F[(i)$-gH]-differentiable, or

$$
u(t)=\Lambda_{2} \oplus u_{0} t \ominus \frac{-1}{\Gamma(q)} \int_{0}^{t}(t-s)^{q-1} f(s, u(s)) d s, \quad t \in[0, T]
$$

if $\lambda \in(0,1), u$ is (i)-gH-differentiable and $u_{g H}^{\prime}$ is $C F[(i i)$-gH]-differentiable, or

$$
u(t)=\Lambda_{1} \ominus(-1)\left[u_{0} t \oplus \frac{1}{\Gamma(q)} \int_{0}^{t}(t-s)^{q-1} f(s, u(s)) d s\right], \quad t \in[0, T]
$$

if $\lambda \in(1,+\infty), u$ is (ii)-gH-differentiable and $u_{g H}^{\prime}$ is $C F[(i)$-gH]-differentiable, or 
(K4)

$$
u(t)=\Lambda_{2} \ominus(-1)\left[u_{0} t \ominus \frac{-1}{\Gamma(q)} \int_{0}^{t}(t-s)^{q-1} f(s, u(s)) d s\right], t \in[0, T]
$$

if $\lambda \in(1,+\infty), u$ is (ii)-gH-differentiable and $u_{g H}^{\prime}$ is ${ }^{C F}[(i i)$-gH]-differentiable,

where

$$
\Lambda_{1}=\frac{\lambda}{1-\lambda}\left(u_{0} T \oplus \frac{1}{\Gamma(q)} \int_{0}^{T}(T-s)^{q-1} f(s, u(s)) d s\right)
$$

and

$$
\Lambda_{2}=\frac{\lambda}{1-\lambda}\left(u_{0} T \ominus \frac{-1}{\Gamma(q)} \int_{0}^{T}(T-s)^{q-1} f(s, u(s)) d s\right) .
$$

Proof. Let $u(0)=u_{1} \in \mathbb{E}_{c}^{1}$. Then, the proof of this lemma is just divided into two cases for the sake of clarity.

Case 1. $\lambda \in(0,1)$. Firstly, we consider situation (K1). By the Lemma 2.10, with initial conditions $u(0)=u_{1}$ and $u_{g H}^{\prime}(0)=u_{0}$, equation (1.1) is equivalent to the fuzzy integral equation

$$
u(t)=u_{1} \oplus u_{0} t \oplus \frac{1}{\Gamma(q)} \int_{0}^{t}(t-s)^{q-1} f(s, u(s)) d s, \quad t \in[0, T] .
$$

To meet boundary value condition $u(0)=\lambda u(T)$, equation (3.3) must satisfy

$$
\lambda\left(u_{1} \oplus u_{0} T \oplus \frac{1}{\Gamma(q)} \int_{0}^{T}(T-s)^{q-1} f(s, u(s)) d s\right)=u_{1} .
$$

By Lemma 2.2, equation (3.4) has solution

$$
u_{1}=\frac{\lambda}{1-\lambda}\left(u_{0} T \oplus \frac{1}{\Gamma(q)} \int_{0}^{T}(T-s)^{q-1} f(s, u(s)) d s\right)=\Lambda_{1} .
$$

By substituting $\Lambda_{1}$ into (3.3), we get (3.1). Conversely, equation (3.1) apparently satisfies (1.1) as well as condition (1.2). For situation (K2), the proof is quite similar to situation (K1) and so is omitted.

Case 2. $\lambda \in(1,+\infty)$. Firstly, we consider situation (K3). By the Lemma 2.10, with initial conditions $u(0)=u_{1}$ and $u_{g H}^{\prime}(0)=u_{0}$, equation (1.1) is equivalent to the fuzzy integral equation

$$
u(t)=u_{1} \ominus(-1)\left[u_{0} t \oplus \frac{1}{\Gamma(q)} \int_{0}^{t}(t-s)^{q-1} f(s, u(s)) d s\right], t \in[0, T] .
$$

To meet boundary value condition $u(0)=\lambda u(T)$, equation (3.5) must satisfy

$$
\lambda\left(u_{1} \ominus(-1)\left[u_{0} T \oplus \frac{1}{\Gamma(q)} \int_{0}^{T}(t-s)^{q-1} f(s, u(s)) d s\right]\right)=u_{1},
$$

which is equivalent to

$$
u_{1}=\frac{1}{\lambda} u_{1} \oplus(-1)\left[u_{0} T \oplus \frac{1}{\Gamma(q)} \int_{0}^{T}(t-s)^{q-1} f(s, u(s)) d s\right] .
$$

By Lemma 2.2, equation (3.6) has solution

$$
u_{1}=\frac{\lambda}{1-\lambda}\left(u_{0} T \oplus \frac{1}{\Gamma(q)} \int_{0}^{T}(T-s)^{q-1} f(s, u(s)) d s\right)=\Lambda_{1} .
$$

By substituting $\Lambda_{1}$ into (3.5), we get (3.2). Conversely, equation (3.2) apparently satisfies (1.1) as well as condition (1.2). For situation (K4), the proof is quite similar to situation (K3) and so is omitted. The proof is completed. 
Corollary 3.1. If $\lambda=0$, boundary value problems (1.1)-(1.2) degenerate into the following initial value problem

$$
\left\{\begin{array}{l}
{ }_{g H}^{C} \mathfrak{D}_{*}^{q} u(t)=f(t, u(t)), \\
u(0)=\widehat{0}, u_{g H}^{\prime}(0)=u_{0} \in \mathbb{E}^{1}
\end{array}\right.
$$

and it is equivalent to

$$
u(t)=u_{0} t \oplus \frac{1}{\Gamma(q)} \int_{0}^{t}(t-s)^{q-1} f(s, u(s)) d s
$$

if $u$ is (i)- $g H$-differentiable and $u_{g H}^{\prime}$ is $C F[(\mathrm{i})-g H]$-differentiable, or

$$
u(t)=u_{0} t \ominus \frac{-1}{\Gamma(q)} \int_{0}^{t}(t-s)^{q-1} f(s, u(s)) d s
$$

if $u$ is (i)- $g H$-differentiable and $u_{g H}^{\prime}$ is $C F[($ ii)- $g H]$-differentiable.

In the following, we impose growth conditions on $f$ which allow us to establish some results for existence and uniqueness of solution to boundary value problems (1.1)-(1.2).

Theorem 3.1. Suppose that $\lambda \in(0,1)$ and

(H1) $f:[0, T] \times \mathbb{E}_{c}^{1} \rightarrow \mathbb{E}_{c}^{1} \backslash \mathbb{R}$ is a given continuous and compact fuzzy-valued function;

(H2) $M:=\sup _{(t, u) \in[0, T] \times \mathbb{E}_{c}^{1}} d(f(t, u), \widehat{0})<+\infty$.

Then boundary value problems (1.1)-(1.2) have at least one ${ }^{C F}\left[(i-i)\right.$-gH]-differentiable solution or ${ }^{C F}[(i$ ii)-gH]-differentiable solution.

Proof. Let $S=C^{\mathbb{E}}\left([0, T], \mathbb{E}_{c}^{1}\right)$. Then $S$ is a semi-linear Banach space having the cancellation property with respect to $\oplus$. Define a set $B \subset S$ by

$$
B=\{u \in S \mid D(u, \widehat{0}) \leq R\}
$$

for some fixed $R>0$. It is easy to know that $B$ is a nonempty, closed, bounded and convex subset of $S$. Choose $R=\frac{T}{1-\lambda} d\left(u_{0}, \widehat{0}\right)+\frac{M T^{q}}{(1-\lambda) \Gamma(q+1)}+1$. Then, the proof of this theorem is just divided into two cases for the sake of clarity.

Case 1. $u$ is (i)- $g H$-differentiable and $u_{g H}^{\prime}$ is $C F[(\mathrm{i})-g H]$-differentiable. From situation (K1) of Lemma 3.1 , boundary value problems (1.1)-(1.2) are equivalent to integral equation

$$
u(t)=\Lambda_{1} \oplus u_{0} t \oplus \frac{1}{\Gamma(q)} \int_{0}^{t}(t-s)^{q-1} f(s, u(s)) d s, \quad t \in[0, T] .
$$

Then, we define the operator $A_{1}: B \rightarrow S$ by

$$
\left(A_{1} u\right)(t):=\Lambda_{1} \oplus u_{0} t \oplus \frac{1}{\Gamma(q)} \int_{0}^{t}(t-s)^{q-1} f(s, u(s)) d s, \quad t \in[0, T]
$$

In order to apply Lemma 2.11, we separate the proof into the following three steps. 
Step 1. $A_{1}$ maps $B$ into itself. For any $u \in B$ and $t \in[0, T]$, from Lemmas 2.3 and 2.6 as well as (H2), we have

$$
\begin{aligned}
d\left(A_{1} u(t), \widehat{0}\right)= & d\left(\Lambda_{1} \oplus u_{0} t \oplus \frac{1}{\Gamma(q)} \int_{0}^{t}(t-s)^{q-1} f(s, u(s)) d s, \widehat{0}\right) \\
\leq & \frac{\lambda}{1-\lambda} d\left(u_{0} T, \widehat{0}\right)+\frac{\lambda}{(1-\lambda) \Gamma(q)} d\left(\int_{0}^{T}(T-s)^{q-1} f(s, u(s)) d s, \widehat{0}\right) \\
& +d\left(u_{0} t, \widehat{0}\right)+\frac{1}{\Gamma(q)} d\left(\int_{0}^{t}(t-s)^{q-1} f(s, u(s)) d s, \widehat{0}\right) \\
\leq & \frac{\lambda}{1-\lambda} d\left(u_{0} T, \widehat{0}\right)+\frac{\lambda}{(1-\lambda) \Gamma(q)} \int_{0}^{T}(T-s)^{q-1} d(f(s, u(s)), \widehat{0}) d s \\
& +d\left(u_{0} t, \widehat{0}\right)+\frac{1}{\Gamma(q)} \int_{0}^{t}(t-s)^{q-1} d(f(s, u(s)), \widehat{0}) d s \\
\leq & \frac{\lambda}{1-\lambda} d\left(u_{0} T, \widehat{0}\right)+\frac{\lambda M}{(1-\lambda) \Gamma(q)} \int_{0}^{T}(T-s)^{q-1} d s+d\left(u_{0} t, \widehat{0}\right)+\frac{M}{\Gamma(q)} \int_{0}^{t}(t-s)^{q-1} d s \\
= & \frac{\lambda}{1-\lambda} d\left(u_{0} T, \widehat{0}\right)+\frac{\lambda M}{(1-\lambda) \Gamma(q+1)} T^{q}+d\left(u_{0} t, \widehat{0}\right)+\frac{M}{\Gamma(q+1)} t^{q} \\
\leq & \frac{T}{1-\lambda} d\left(u_{0}, \widehat{0}\right)+\frac{M T^{q}}{(1-\lambda) \Gamma(q+1)} \leq R .
\end{aligned}
$$

It follows that $D\left(A_{1} u, \widehat{0}\right) \leq R$. Thus, $A_{1}$ maps $B$ into itself.

Step 2. $A_{1}$ is a continuous operator on $B$. For this, let $u_{n} \rightarrow u(n \rightarrow \infty)$ in $B$. From Lemmas 2.3 and 2.6, we have

$$
\begin{aligned}
& d\left(A_{1} u_{n}(t), A_{1} u(t)\right) \\
\leq & \frac{\lambda}{(1-\lambda) \Gamma(q)} d\left(\int_{0}^{T}(T-s)^{q-1} f\left(s, u_{n}(s)\right) d s, \int_{0}^{T}(T-s)^{q-1} f(s, u(s)) d s\right) \\
& +\frac{1}{\Gamma(q)} d\left(\int_{0}^{t}(t-s)^{q-1} f\left(s, u_{n}(s)\right) d s, \int_{0}^{t}(t-s)^{q-1} f(s, u(s)) d s\right) \\
\leq & \frac{\lambda}{(1-\lambda) \Gamma(q)} \int_{0}^{T}(T-s)^{q-1} d\left(f\left(s, u_{n}(s)\right), f(s, u(s))\right) d s \\
& +\frac{1}{\Gamma(q)} \int_{0}^{t}(t-s)^{q-1} d\left(f\left(s, u_{n}(s)\right), f(s, u(s))\right) d s \\
\leq & \left.\frac{\lambda T^{q}}{(1-\lambda) \Gamma(q+1)} \sup _{t \in[0, T]} d\left(f\left(t, u_{n}(t)\right), f(t, u(t))\right)+\frac{t^{q}}{\Gamma(q+1)} \sup _{t \in[0, T]} d\left(f\left(t, u_{n}(t)\right), f(t, u(t))\right)\right) \\
\leq & \frac{T^{q}}{(1-\lambda) \Gamma(q+1)} \sup _{t \in[0, T]} d\left(f\left(t, u_{n}(t)\right), f(t, u(t))\right) .
\end{aligned}
$$

According to (H1), $f$ is continuous on $[0, T] \times \mathbb{E}_{c}^{1}$. So, $\sup _{t \in[0, T]} d\left(f\left(t, u_{n}(t)\right), f(t, u(t))\right) \rightarrow 0$ when $n \rightarrow \infty$. We get $D\left(A_{1} u_{n}, A_{1} u\right) \rightarrow 0$ when $n \rightarrow \infty$. So $A_{1}$ is a continuous operator on $B$.

Step 3. $A_{1}(B)$ is relatively compact in $C^{\mathbb{E}}\left([0, T], \mathbb{E}_{c}^{1}\right)$. According to Lemma 2.4 , we just need to prove

(i) $A_{1}(B)$ is equicontinuous;

(ii) for every $t \in[0, T]$, the set $A_{1}(B)(t)$ is relatively compact in $\mathbb{E}_{c}^{1}$. 
Firstly, we verify the equicontinuity of $A_{1}(B)$. Let $t_{1}, t_{2} \in[0, T], t_{1}<t_{2}$ and $u \in B$. From Lemmas 2.3 and 2.6 as well as (H2), we get

$$
\begin{aligned}
& d\left(A_{1} u\left(t_{2}\right), A_{1} u\left(t_{1}\right)\right) \\
= & d\left(u_{0} t_{2} \oplus \frac{1}{\Gamma(q)} \int_{0}^{t_{2}}\left(t_{2}-s\right)^{q-1} f(s, u(s)) d s, u_{0} t_{1} \oplus \frac{1}{\Gamma(q)} \int_{0}^{t_{1}}\left(t_{2}-s\right)^{q-1} f(s, u(s)) d s\right) \\
\leq & d\left(\frac{1}{\Gamma(q)} \int_{0}^{t_{2}}\left(t_{2}-s\right)^{q-1} f(s, u(s)) d s, \frac{1}{\Gamma(q)} \int_{0}^{t_{1}}\left(t_{2}-s\right)^{q-1} f(s, u(s)) d s\right)+d\left(u_{0} t_{2}, u_{0} t_{1}\right) \\
= & \frac{1}{\Gamma(q)} d\left(\int_{0}^{t_{1}}\left(t_{2}-s\right)^{q-1} f\left(s, u_{n}(s)\right) d s \oplus \int_{t_{1}}^{t_{2}}\left(t_{2}-s\right)^{q-1} f\left(s, u_{n}(s)\right) d s, \int_{0}^{t_{1}}\left(t_{1}-s\right)^{q-1} f(s, u(s)) d s\right) \\
& +d\left(u_{0} t_{2}, u_{0} t_{1}\right) \\
\leq & \frac{1}{\Gamma(q)} \int_{0}^{t_{1}}\left[\left(t_{2}-s\right)^{q-1}-\left(t_{1}-s\right)^{q-1}\right] d(f(s, u(s)), \widehat{0}) d s+\frac{1}{\Gamma(q)} \int_{t_{1}}^{t_{2}}\left(t_{2}-s\right)^{q-1} d(f(s, u(s)), \widehat{0}) d s \\
& +d\left(u_{0} t_{2}, u_{0} t_{1}\right) \\
\leq & \frac{M}{\Gamma(q)} \int_{0}^{t_{1}}\left[\left(t_{2}-s\right)^{q-1}-\left(t_{1}-s\right)^{q-1}\right] d s+\frac{M}{\Gamma(q)} \int_{t_{1}}^{t_{2}}\left(t_{2}-s\right)^{q-1} d s+\left(t_{2}-t_{1}\right) d\left(u_{0}, \widehat{0}\right) \\
= & \frac{M}{\Gamma(q+1)}\left[2\left(t_{2}-t_{1}\right)^{q}+\left(t_{2}^{q}-t_{1}^{q}\right)\right]+\left(t_{2}-t_{1}\right) d\left(u_{0}, \widehat{0}\right) .
\end{aligned}
$$

This implies that $d\left(A_{1} u\left(t_{2}\right), A_{1} u\left(t_{1}\right)\right) \rightarrow 0$ when $\left|t_{2}-t_{1}\right| \rightarrow 0$. Hence $A_{1}(B)$ is an equicontinuous subset of $C^{\mathbb{E}}\left([0, T], \mathbb{E}_{c}^{1}\right)$.

Secondly, we prove that $A_{1}(B)(t)$ is relatively compact in $\mathbb{E}_{c}^{1}$. On the one hand, due to the relative compactness of $f([0, T] \times B)$, there exists compact set $\widetilde{K}$ such that $[f(t, u)]^{0} \subseteq \widetilde{K}$ for all $(t, u) \in[0, T] \times B$. So

$$
\begin{aligned}
{\left[A_{1}(B)(t)\right]^{0}=} & {\left[\Lambda_{1} \oplus u_{0} t \oplus \frac{1}{\Gamma(q)} \int_{0}^{t}(t-s)^{q-1} f(s, u(s)) d s\right]^{0} } \\
= & \frac{\lambda T}{1-\lambda}\left[u_{0}\right]^{0}+\frac{\lambda}{(1-\lambda) \Gamma(q)} \int_{0}^{T}(T-s)^{q-1}[f(s, u(s))]^{0} d s \\
& +t\left[u_{0}\right]^{0}+\frac{1}{\Gamma(q)} \int_{0}^{t}(t-s)^{q-1}[f(s, u(s))]^{0} d s \\
\subseteq & {\left[\frac{\lambda}{(1-\lambda) \Gamma(q)} \int_{0}^{T}(T-s)^{q-1} d s+\frac{1}{\Gamma(q)} \int_{0}^{t}(t-s)^{q-1} d s\right] \widetilde{K}+\frac{T}{1-\lambda}\left[u_{0}\right]^{0} } \\
= & {\left[\frac{\lambda T^{q}}{(1-\lambda) \Gamma(q+1)}+\frac{t^{q}}{\Gamma(q+1)}\right] \widetilde{K}+\frac{T}{1-\lambda}\left[u_{0}\right]^{0} . }
\end{aligned}
$$

Therefore, there exists a compact set $K_{0} \subseteq \mathbb{R}$ such that $\left[A_{1}(B)(t)\right]^{0} \subseteq K_{0}$, that is, $A_{1}(B)(t)$ is compactsupported for every $t \in[0, T]$. On the other hand, fixing $t \in[0, T]$, we have $A_{1}(B)(t) \in \mathbb{E}_{c}^{1}$. If $y \in A_{1}(B)(t)$, then $y=A_{1}(u)(t)$ for some $u \in B$. So, we have

$$
[y]^{\alpha}=\Lambda_{1} \oplus t\left[u_{0}\right]^{\alpha} \oplus \frac{1}{\Gamma(q)} \int_{0}^{t}(t-s)^{q-1}[f(s, u(s))]^{\alpha} d s, \quad \alpha \in[0,1]
$$

By Lemma 2.5, we know that $f([0, T] \times B)$ is level-equicontinuous. Hence for any $\varepsilon>0$, there exists $\delta>0$, such that

$$
d_{H}\left([f(t, u)]^{\alpha},[f(t, u)]^{\beta}\right) \leq \frac{(1-\lambda) \Gamma(q+1) \varepsilon}{2 T^{q}} \text { for all }(t, u) \in[0, T] \times B
$$


and $d_{H}\left(\left[u_{0}\right]^{\alpha},\left[u_{0}\right]^{\beta}\right) \leq \frac{(1-\lambda) \varepsilon}{2 T}$ when $|\alpha-\beta|<\delta$. So for all $|\alpha-\beta|<\delta$ and $(t, u) \in[0, T] \times B$, one gets

$$
\begin{aligned}
& d_{H}\left([y]^{\alpha},[y]^{\beta}\right) \\
= & d_{H}\left(\left[A_{1} u(t)\right]^{\alpha},\left[A_{1} u(t)\right]^{\beta}\right) \\
\leq & \frac{\lambda T}{1-\lambda} d_{H}\left(\left[u_{0}\right]^{\alpha},\left[u_{0}\right]^{\beta}\right)+\frac{\lambda}{(1-\lambda) \Gamma(q)} \int_{0}^{T}(T-s)^{q-1} d_{H}\left([f(s, u(s))]^{\alpha},[f(s, u(s))]^{\beta}\right) d s \\
& +t d_{H}\left(\left[u_{0}\right]^{\alpha},\left[u_{0}\right]^{\beta}\right)+\frac{1}{\Gamma(q)} \int_{0}^{t}(t-s)^{q-1} d_{H}\left([f(s, u(s))]^{\alpha},[f(s, u(s))]^{\beta}\right) d s \\
\leq & \frac{\lambda T}{1-\lambda} d_{H}\left(\left[u_{0}\right]^{\alpha},\left[u_{0}\right]^{\beta}\right)+\frac{\lambda T^{q}}{(1-\lambda) \Gamma(q+1)} \cdot \frac{(1-\lambda) \Gamma(q+1) \varepsilon}{2 T^{q}} \\
& +t d_{H}\left(\left[u_{0}\right]^{\alpha},\left[u_{0}\right]^{\beta}\right)+\frac{t^{q}}{\Gamma(q+1)} \cdot \frac{(1-\lambda) \Gamma(q+1) \varepsilon}{2 T^{q}} \leq \varepsilon,
\end{aligned}
$$

which means $A_{1}(B)(t)$ is level-equicontinuous in $\mathbb{E}_{c}^{1}$ on $[0,1]$ for each $t \in[0, T]$. Therefore, $A_{1}(B)(t)$ is a relatively compact subset of $\mathbb{E}_{c}^{1}$ from Lemma 2.5. Then according to Lemma 2.4 , we obtain that $A_{1}(B)$ is relatively compact in $C^{\mathbb{E}}\left([0, T], \mathbb{E}_{c}^{1}\right)$.

Through the above elaboration, we conclude that $A_{1}$ is a compact operator. By Lemma 2.11, it follows that $A_{1}$ has at least one fixed point in $B$. Equivalently, there is at least one ${ }^{C F}[(\mathrm{i}-\mathrm{i})-g H]$-differentiable solution to boundary value problems (1.1)-(1.2).

Case 2. $u$ is (i)- $g H$-differentiable and $u_{g H}^{\prime}$ is $C F[(\mathrm{ii})-g H]$-differentiable. From situation (K2) of Lemma 3.1 , boundary value problems (1.1)-(1.2) are equivalent to integral equation

$$
u(t)=\Lambda_{2} \oplus u_{0} t \ominus \frac{-1}{\Gamma(q)} \int_{0}^{t}(t-s)^{q-1} f(s, u(s)) d s, \quad t \in[0, T] .
$$

Then, we define the operator $A_{2}: B \rightarrow S$ by

$$
\left(A_{2} u\right)(t)=\Lambda_{2} \oplus u_{0} t \ominus \frac{-1}{\Gamma(q)} \int_{0}^{t}(t-s)^{q-1} f(s, u(s)) d s, \quad t \in[0, T] .
$$

For any $u \in B$ and $t \in[0, T]$, from Lemmas 2.3 and 2.6 as well as (H2), we have

$$
\begin{aligned}
d\left(A_{2} u(t), \widehat{0}\right)= & d\left(\Lambda_{2} \oplus u_{0} t \ominus \frac{-1}{\Gamma(q)} \int_{0}^{t}(t-s)^{q-1} f(s, u(s)) d s, \widehat{0}\right) \\
\leq & \frac{\lambda}{1-\lambda} d\left(u_{0} T, \widehat{0}\right)+\frac{\lambda}{(1-\lambda) \Gamma(q)} d\left(\int_{0}^{T}(T-s)^{q-1} f(s, u(s)) d s, \widehat{0}\right) \\
& +d\left(u_{0} t, \widehat{0}\right)+\frac{1}{\Gamma(q)} d\left(\int_{0}^{t}(t-s)^{q-1} f(s, u(s)) d s, \widehat{0}\right) \\
\leq & \frac{T}{1-\lambda} d\left(u_{0}, \widehat{0}\right)+\frac{M T^{q}}{(1-\lambda) \Gamma(q+1)} \leq R
\end{aligned}
$$

which means $A_{2}$ maps $B$ into itself. The remainder of the argument is analogous to that in Case 1 but will not be reproduced here. The proof is completed.

Theorem 3.2. Suppose $\lambda \in(0,1)$ and $f$ satisfies (H1)-(H2) as well as the following condition:

(H3) there exists a constant $L \geq 0$, for all $u, v \in \mathbb{E}_{c}^{1}$, such that

$$
d(f(t, u), f(t, v)) \leq L d(u, v) .
$$

Then boundary value problems (1.1)-(1.2) have a unique ${ }^{C F}\left[(i-i)\right.$-gH]-differentiable solution or ${ }^{C F}[(i$-ii)gH]-differentiable solution on $[0, T]$. 
Proof. Since $\lambda \in(0,1)$ and $f$ satisfies (H1)-(H2), it follows that boundary value problems (1.1)-(1.2) have at least one ${ }^{C F}[(\mathrm{i}-\mathrm{i})-g H]$-differentiable solution or ${ }^{C F}[(\mathrm{i}-\mathrm{ii})-g H]$-differentiable solution according to Theorem 3.1. In the sequel, we only prove ${ }^{C F}[(\mathrm{i}-\mathrm{i})-g H]$-differentiable case. The proof of the other case is similar.

Suppose there exist two $C F[(\mathrm{i}-\mathrm{i})-g H]$-differentiable solutions $u$ and $v$ to boundary value problems (1.1)-(1.2). Then, for all $t \in[0, T]$, we have

$$
\begin{aligned}
d(u(t), v(t))= & d\left(A_{1} u(t),\left(A_{1} v(t)\right)\right. \\
\leq & \frac{\lambda}{(1-\lambda) \Gamma(q)} d\left(\int_{0}^{T}(T-s)^{q-1} f(s, u(s)) d s, \int_{0}^{T}(T-s)^{q-1} f(s, v(s)) d s\right) \\
& +\frac{1}{\Gamma(q)} d\left(\int_{0}^{t}(t-s)^{q-1} f(s, u(s)) d s, \int_{0}^{t}(t-s)^{q-1} f(s, v(s)) d s\right) \\
\leq & \frac{\lambda}{(1-\lambda) \Gamma(q)} \int_{0}^{T}(T-s)^{q-1} d(f(s, u(s)), f(s, v(s))) d s \\
& +\frac{1}{\Gamma(q)} \int_{0}^{t}(t-s)^{q-1} d(f(s, u(s)), f(s, v(s))) d s \\
\leq & {\left[\frac{\lambda L}{(1-\lambda) \Gamma(q)}+\frac{L}{\Gamma(q)}\right] \int_{0}^{T}(T-s)^{q-1} d(u(s), v(s)) d s . }
\end{aligned}
$$

Therefore, we obtain $u(t)=v(t)$ for all $t \in[0, T]$ according to Lemma 2.12. Hence the solution is unique. The proof is completed.

Corollary 3.2. Suppose $\lambda=0$. For the initial value problem (3.7), we also have corresponding results if it satisfies the conditions of Theorem 3.1 or 3.2.

Theorem 3.3. Suppose that $\lambda \in(1,+\infty)$ and right-hand side function $f$ satisfies (H1)-(H2). Then boundary value problems (1.1)-(1.2) have at least one ${ }^{C F}\left[(i i-i)\right.$-gH]-differentiable solution or ${ }^{C F}[(i i$-ii)gH]-differentiable solution.

Proof. For the set $B$ defined as (3.8), choose $R=\frac{(2 \lambda-1) T}{\lambda-1} d\left(u_{0}, \widehat{0}\right)+\frac{(2 \lambda-1) M T^{q}}{(\lambda-1) \Gamma(q+1)}+1$. If $u$ is (ii)-gHdifferentiable and $u_{g H}^{\prime}$ is ${ }^{C F}[(\mathrm{i})-g H]$-differentiable, then we consider the operator $A_{3}: B \rightarrow S$ defined by

$$
\left(A_{3} u\right)(t)=\Lambda_{1} \ominus(-1)\left[u_{0} t \oplus \frac{1}{\Gamma(q)} \int_{0}^{t}(t-s)^{q-1} f(s, u(s)) d s\right] .
$$

If $u$ is (ii)- $g H$-differentiable and $u_{g H}^{\prime}$ is $C F[($ ii)- $g H]$-differentiable, then can we consider the operator operator $A_{4}: B \rightarrow S$ defined by

$$
\left(A_{4} u\right)(t)=\Lambda_{2} \ominus(-1)\left[u_{0} t \ominus \frac{-1}{\Gamma(q)} \int_{0}^{t}(t-s)^{q-1} f(s, u(s)) d s\right] .
$$

The remainder of the argument is analogous to the proof of Theorem 3.1. The proof is completed.

Similar to Theorem 3.2, we can get the following uniqueness results.

Theorem 3.4. Suppose $\lambda \in(1,+\infty)$ and $f$ satisfies (H1)-(H3). Then boundary value problems (1.1)-(1.2) have a unique ${ }^{C F}\left[(i i-i)\right.$-gH]-differentiable solution or ${ }^{C F}[(i i-i i)$-gH]-differentiable solution on $[0, T]$.

\section{Example}

In this section, we will present an example to illustrate our main results. Consider the following boundary value problem

$$
\left\{\begin{array}{l}
{ }_{g H}^{C} \mathfrak{D}_{*}^{\frac{3}{2}} u(t)=\sin u(t) \oplus \eta e^{-t}, \quad 0<t<1, \\
u(0)=0.75 u(1), u_{g H}^{\prime}(0)=\widehat{0},
\end{array}\right.
$$


where fuzzy sinusoidal function $\sin u(t)$ is obtained by Zadeh's extension principle, and fuzzy constants $\eta=(0.5,1,1.5)$ and $\widehat{0}=(0,0,0)$ are two symmetric triangular fuzzy numbers. Let $f(t, u)=\sin u+\eta e^{-t}$. Apparently, $f$ is a continuous and compact fuzzy-valued function. For $t \in[0,1]$, from the definition of $d(u, v)$ and Lemma 2.2 , we can easily get

$$
\begin{aligned}
M & =\sup _{(t, u) \in[0,1] \times \mathbb{E}_{c}^{1}} d(f(t, u), \widehat{0}) \\
& =\sup _{(t, u) \in[0,1] \times \mathbb{E}_{c}^{1}} d\left(\sin u(t) \oplus \eta e^{-t}, \widehat{0}\right) \\
& \leq \sup _{(t, u) \in[0,1] \times \mathbb{E}_{c}^{1}} d(\sin u(t), \widehat{0})+\sup _{(t, u) \in[0,1] \times \mathbb{E}_{c}^{1}} d\left(\eta e^{-t}, \widehat{0}\right) \\
& \leq \sup _{(t, u) \in[0,1] \times \mathbb{E}_{c}^{1}} d(\sin u(t), \widehat{0})+\sup _{(t, u) \in[0,1] \times \mathbb{E}_{c}^{1}} d(\eta, \widehat{0}) \\
& =1+1.5=2.5 .
\end{aligned}
$$

So, we take $R=\frac{2.5}{0.25 \Gamma(2.5)}+1 \approx 8.5225$. By Theorem 3.1, the boundary value problem (4.1) has at least one ${ }^{C F}[(\mathrm{i}-\mathrm{i})-g H]$-differentiable solution. At the same time, the Hukuhara difference $u_{0} t \ominus \frac{-1}{\Gamma(q)} \int_{0}^{t}(t-$ $s)^{q-1} f(s, u(s)) d s$ does not exist for any $t \in(0,1]$. It means that problem (4.1) has no $C F[(\mathrm{i}-\mathrm{ii})-g H]$ differentiable solution. In addition, due to

$$
\begin{aligned}
d(f(t, u), f(t, v)) & =d\left(\sin u \oplus \eta e^{-t}, \sin v \oplus \eta e^{-t}\right) \\
& =d(\sin u, \sin v) \\
& \leq d(u, v)
\end{aligned}
$$

for all $u, v \in \mathbb{E}^{1}$, we know the ${ }^{C F}[(\mathrm{i}-\mathrm{i})$ - $g H]$-differentiable solution is unique by Theorem 3.2.

\section{Example}

The most significant part of this study is our research object. This work regarding FFDEs with order $q \in(1,2]$ is novel. Another important part is that some other kinds of fuzzy boundary value problems can also be made under the approach similarly to the above mentioned procedure. The authors of this article strongly believe that this article will also motivate some physicists, engineers and scholars to make further researches in this area. In this respect, there may be other extended work.

Acknowledgments. This research is supported by the Natural Science Foundation of China (61374074, 11571202), and supported by Shandong Provincial Natural Science Foundation (ZR2016AM17).

\section{References}

1. A. Khastan, J. Nieto, "Periodic boundary value problems for first-order linear differential equations with uncertainty under generalized differentiability," Information Sciences, vol. 222, pp. 544-558, 2013.

2. H. Wang, "Monotone iterative method for boundary value problems of fuzzy differential equations," Journal of Intelligent and Fuzzy Systems, vol. 30, pp. 831-843, 2016.

3. T. Allahviranloo, Z. Gouyandeh, A. Armand, A. Hasanoglu, "On fuzzy solutions for heat equation based on generalized Hukuhara differentiability," Fuzzy Sets and Systems, vol. 265, pp. 1-23, 2015.

4. M. Guo, X. Xue, R. Li, "Impulsive functional differential inclusions and fuzzy population models," Fuzzy Sets and Systems, vol. 138, pp. 601-615, 2003.

5. R. Jafelice, L. Barros, R. Bassanez, F. Gomide, "Fuzzy modeling in symptomatic HIV virus infected population," Bulletin of Mathematical Biology, vol. 66, pp. 1597-1620, 2004. 
6. A. Ahmadian, S. Salahshour, D. Baleanu, H. Amirkhani, R. Yunus, "Tau method for the numerical solution of a fuzzy fractional kinetic model and its application to the oil palm frond as a promising source of xylose," Journal of Computational Physics, vol. 294, pp. 562-584, 2015.

7. S. Paul, S. Mondal, P. Bhattacharya, "Discussion on fuzzy quota harvesting model in fuzzy environment: fuzzy differential equation approach," Modeling Earth Systems and Environment, vol. 2, pp. 1-15, 2016.

8. R. Agarwal, V. Lakshmikantham, J. Nieto, "On the concept of solution for fractional differential equations with uncertainty," Nonlinear Analysis, vol. 72, pp. 2859-2862, 2010.

9. R. Agarwal, S. Arshad, D. O'Regan, V. Lupulescu, "A Schauder fixed point theorem in semilinear spaces and applications," Fixed Point Theory and Applications, vol. 306, pp. 1-13, 2013.

10. A. Khastan, J. Nieto, R. Rodríguez-López, "Schauder fixed-point theorem in semilinear spaces and its application to fractional differential equations with uncertainty," Fixed Point Theory and Applications, vol. 21, pp. 1-14, 2014.

11. T. Allahviranloo, A. Armand, Z. Gouyandeh, "Fuzzy fractional differential equations under generalized fuzzy Caputo derivative," Journal of Intelligent and Fuzzy Systems, vol. 26, pp. 1481-1490, 2014.

12. V. Lupulescu, "Fractional calculus for interval-valued functions," Fuzzy Sets and Systems, vol. 265, pp. 63-85, 2015 .

13. V. Hgo, "Fuzzy fractional functional integral and differential equations," Fuzzy Sets and Systems, vol. 280, pp. 58-90, 2015.

14. N. Hoa, "Fuzzy fractional functional differential equations under Caputo gH-differentiability," Communications in Nonlinear Science and Numerical Simulation, vol. 22, pp. 1134-1157, 2015.

15. S. Salahshour, A. Ahmadian, N. Senu, D. Baleanu, P. Agarwal, "On analytical solutions of the fractional differential equation with uncertainty: application to the Basset problem," Entropy, vol. 17, pp. 885-902, 2015.

16. P. Diamond, P. Kloeden, Metric Spaces of Fuzzy Sets: Theory and Applications, World Scientific, Singapore, 1994.

17. V. Lakshmikantham, R. Mohapatra, Theory of Fuzzy Differential Equations and Inclusions, Taylor and Francis, London, 2003.

18. V. Lakshmikantham, B. Gnana, D. Vasundhara, Theory of Set Differential Equations in Metric Spaces, Cambridge Scientific Publishers, 2006.

19. L. Gomes, L. Barros, B. Bede, Fuzzy Differential Equations in Various Approaches, Springer International Publishing, 2015.

20. G. Anastassiou, S. Gal, "On a fuzzy trigonometric approximation theorem of Weierstrass-type," The Journal of Fuzzy Mathematics, vol. 3, pp. 701-708, 2001.

21. M. Puri, D. Ralescu, "Differentials of fuzzy functions," Journal of Mathematical Analysis and Applications, vol. 91, pp. 552-558, 1983.

22. H. Román-Flores, M. Rojas-Medar, "Embedding of level-continuous fuzzy sets on Banach spaces," Information Sciences, vol. 114, pp. 227-247, 2002.

23. O. Kaleva, "Fuzzy differential equations," Fuzzy Sets and Systems, vol. 24, pp. 301-317, 1987.

24. R. Agarwal, S. Arshad, D. O'Regan, V. Lupulescu, "Fuzzy fractional integral equations under compactness type condition," Fractional Calculus and Applied Analysis, vol. 15, pp. 572-590, 2012.

25. L. Stefanini, "A generalization of Hukuhara difference and division for interval and fuzzy arithmetic," Fuzzy Sets and Systems, vol. 161, pp. 1564-1584, 2010.

26. B. Bede, L. Stefanini, "Generalized differentiability of fuzzy-valued functions," Fuzzy Sets and Systems, vol. 230, pp. 119-141, 2013.

27. H. Ye, J. Gao, Y. Ding, "A generalized Gronwall inequality and its application to a fractional differential equation," Journal of Mathematical Analysis and Applications, vol. 328, pp. 1075-1081, 2007. 